\title{
Abscess of the Ligamentum Teres: a Rare Entity
}

\author{
Kaushik Bhattacharya ${ }^{1}\left[\right.$ D $\cdot$ Praveenchandra Reddy $^{2} \cdot$ Prem Dorjee Bhutia $^{2}$
}

Received: 12 July 2021 / Accepted: 17 July 2021 / Published online: 26 July 2021

(c) Association of Surgeons of India 2021

\begin{abstract}
Abscess of the ligamentum teres is one of the rarest causes of acute abdomen and causes severe dilemma and real challenge in clinching the diagnosis. A 69-year-old lady with severe upper abdominal pain with history of gall stones underwent MRI upper abdomen and was diagnosed as abscess of the ligamentum teres. There are only very few reported cases of this entity in the literature.
\end{abstract}

Keywords Ligamentum teres · Abscess · Gallstone

A 69-year-old lady presented with a $4 \mathrm{~cm} \times 3 \mathrm{cmlump}$ in the epigastrium of a week's duration along with features of acute calculus cholecystitis. On examination, a tender localised intra-abdominal mass was palpated in the epigastrium. Ultrasound abdomen detected calculus cholecystitis with a vague space-occupying lesion suspicious of pericholecystic collection with no conclusive definitive diagnosis. MRIof the abdomen revealed loculated thick-walled peripherally enhancing space-occupying lesion with enhancing septations with central hypointense signals on $\mathrm{T} 1$ and mixed to hyperintense signals on $\mathrm{T} 2$ seen in the midline epigastrium along the parietal wall of the peritoneum extending from the umbilicus cranially into the subhepatic space and further insinuating along the ligamentum teres up to the porta hepatis and encasing the common hepatic duct. The diagnosis was abscess of the falciform ligament and ligamentum teres hepatis. The patient underwent conservative management with antibiotics and had a smooth recovery. She will undergo cholecystectomy after the ongoing COVID-19 pandemic (Figs. 1 and 2).

The ligamentum teres hepatis (LTH) is a remnant of the left umbilical vein and runs from the umbilicus to the liver. Abscess of the ligamentum teres hepatis is an extremely rare cause of acute abdomen, and it should be suspected in any sausage-shaped palpable epigastric mass with history of

Kaushik Bhattacharya

kbhattacharya10@yahoo.com

1 CAPFs Composite Hospital BSF Kadamtala, G616, Uttorayon, Matigara, Siliguri 734010, West Bengal, India

2 Neotia Getwel Hospital, Siliguri 734011, West Bengal, India cholecystitis, omphalitis or cholangitis [1]. The pathogenesis happens due to portal pyaemia with subsequently infection to the falciform ligament via the paraumbilical veins. The usual confusion in diagnosis happens due to mistakenly diagnosing the entity as abdominal wall abscess. Abscess of the ligamentum teres hepatis may lead to peritonitis, portal thrombosis, sepsis and external compression leading to intestinal obstruction [2]. The treatment of this condition is usually conservative with antibiotics and ultrasound-guided aspiration/drainage of the abscess. Surgical intervention is only required if there is peritonitis and for elimination of the primary cause like gall bladder stones. There have been only

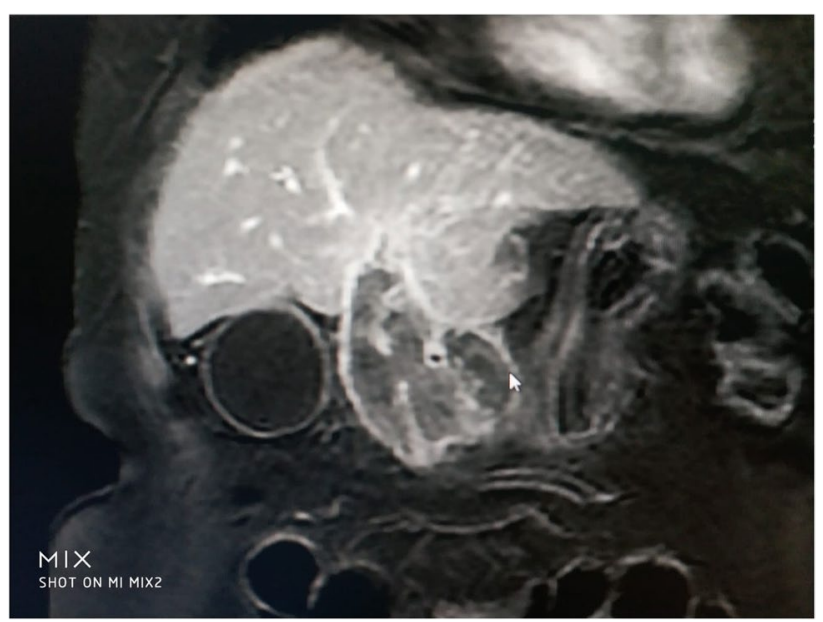

Fig. 1 Abscess of the falciform ligament and ligamentum teres hepatis 


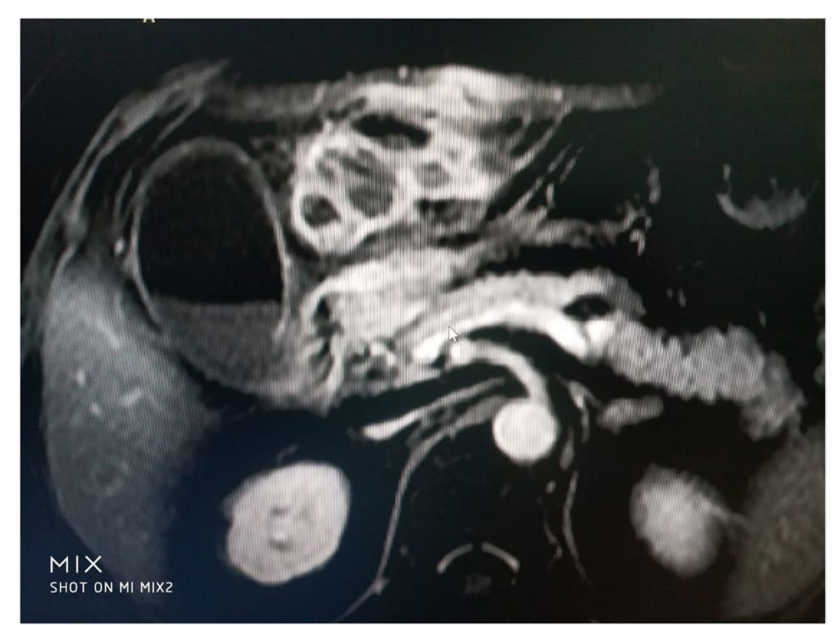

Fig. 2 Gall bladder calculi with the ligamentum teres abscess

3 cases reported with abscess of the ligamentum teres from India till now in the literature, and most probably, this is the fourth case report from India.

\section{Declarations}

Conflict of Interest The authors declare no competing interests.

\section{References}

1. Sen D, Arora V, Sohal RS, Hari PS (2016) The "sausage" abscess: abscess of the ligamentum teres hepatis. BJR Case Rep 2(4):20150139. https://doi.org/10.1259/bjrcr.20150139

2. Warren LR, Chandrasegaram MD, Madigan DJ et al (2012) Falciform ligament abscess from left sided portal pyaemia following malignant obstructive cholangitis. World J Surg Oncol 10:278. https://doi.org/10.1186/1477-7819-10-278

Publisher's Note Springer Nature remains neutral with regard to jurisdictional claims in published maps and institutional affiliations. 